\title{
A Case of Adrenoleucodystrophy: Newer Challenge to Rehabilitation
}

\author{
Newaz $\mathrm{F}^{1}$, Jashimuddin $\mathrm{J}^{1}$, Nuery $\mathrm{N}^{2}$, Uddin $\mathrm{T}^{3}$
}

\begin{abstract}
Adrenoleucodystrophy is a rare, genetic demyelinating disorder. Early onset of disease have rapid progression and worse prognosis. It may be associated with adrenal insufficiency. Not much treatment option as though rehabilitation is mainstream of management till death to reduce disability. We report the case of a 10-year-old boy with progressive weakness of all four limb and speech, swallowing difficulty, whose computerized tomography (CT) and Magnetic resonance imaging (MRI) scans showed unusually florid bilateral abnormalities. MRI showed hyperintensities on parieto-occipital lobe through corpus callosum and some biochemical imbalance on serum. The child was diagnosed as a case of Adrenoleukodystrophy and was presented in a clinical meeting for further managements including medical rehab. As this is a very rare case, it was a challenge to handle such type of patient with a course of combined rehabilitation program and discharged home.
\end{abstract}

Keywords: Adrenoleucodystrophy, rehabilitation

\section{INTRODUCTION}

Adrenoleukodystrophy is a demyelinating disorder of hereditry origin. It is characterized by progressive demyelination of cerebral white matter and adrenal insufficiency. ${ }^{1}$ Adrenoleukodystrophy is an unusual disorder in which progressive diffuse demyelination of the cerebrum is associated with adrenal insufficiency, and which is transmitted as a sex-linked recessive trait. ${ }^{2}$ Most

1. Dr. Fatema Newaz, MBBS, FCPS (Physical Medicine), Consultant at LABAID, Mymensing. E-mil: washimafatin@gmail.com

2. Dr. Jasmine Jashimuddin, MBBS, FCPS course student at Physical Medicine and Rehabilitation, BSMMU, Dhaka

3. Dr. Nuzhat Nuery, MBBS, FCPS course student at Physical Medicine and Rehabilitation, BSMMU, Dhaka

4. Dr. Taslim Uddin, Professor and chairmen, Department of Physical Medicine and Rehabilitation, Bangabandhu Sheikh Mujib Medical University, Shahbag, Dhaka, Bangladesh

*For correspondence common form of ALD is X-linked disorder, it has various presentation and caused by mutations in ABCD1 gene located on $\mathrm{Xq} 28$ which is transmembrane transporter responsible for importation of very long chain fatty acid (VLCFA). Its presentation is highly variable may lead to delayed recognition, attention defecit or hyperactivity disorder in boys and multiple sclerosis in young adult. Most common presentation is severe dementia, visual problem, hearing disturbance, speech \& gait problem, death within few years. Usually patient have adrenal insufficiency at the time of adrenal presentation. ${ }^{3}$ The diagnosis was suggested by clinical and laboratory signs of primary adrenal failure and by neurological signs referable to the degeneration of white matter. Neurological findings usually predominated over clinical stigmata of adrenal failure. ${ }^{4}$ At the course of disease progression, it is rapidly progressible, patient usually reach vegetative state within 10 years after neurological symptom appear. Diagnosis of this disease usually suggested by clinical presentation, biochemical marker, MRI findings. Here a case is reported to focus on its end stage rehabilitation program for

\section{CASE REPORT}

A 10 years old boy comes with uncontrolled fit for last six months. Two years ago, he started having complaints of progressive weakness of all 4 limbs as well as loss of neck control, sitting \& standing balance. Difficulty in swallowing \& unable to talk \& loss of bowel, bladder control for $1^{1 / 2}$ years. According to his parents initially he developed weakness of left upper limb \& kept his left upper limb in flexed position followed by weakness of left lower limb. They also complaint of rapid reduction of school performance $\&$ hand writing. For last 6 months, child is bed ridden, not walking, not responding and not moving any of his limb and difficulty in swallowing solid food \& unable to talk \& makes incomprehensible sound. The patient was born of caesarean section $\&$ post natal period was uneventful. His milestone of development was normal. His parents gave no history of consanguineous of marriage. Physical examination: Height $=137 \mathrm{~cm}$, weight $=25.5 \mathrm{~kg}$, 

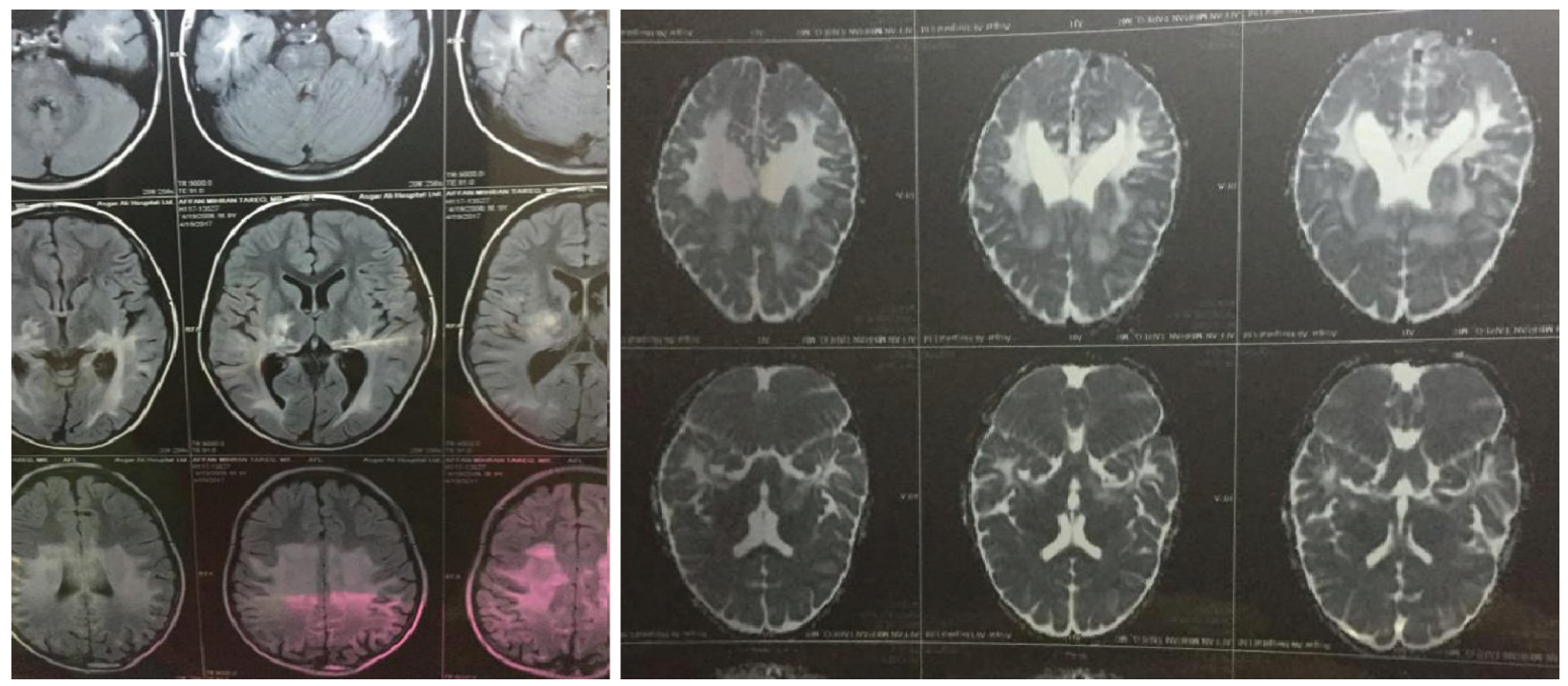

Figure 1: MRI Findings

Vitals-B.P.-120/70 mmHg, pulse-84b.p.m.Both shoulder= sulcus sign present speech $=$ motor aphasia, bulk of the muscles were normal, Tone increased, Power of the muscle were difficult to measure but apparently his weakness were more in hip adductor which was $1 / 5$ on right side and 2/5 on left side, hip Extensor: 2/5 on both, hip Flexor: 2/5 both. Deep tendon Reflex were brisk on both side, bilateral exaggerated planter response.

Results of routine blood count, serum electrolytes, liver function tests, renal function tests and urine analysis were normal. Serum ACTH level was raised $(89.5 \mathrm{pg} / \mathrm{ml}$, Normal range $10-50 \mathrm{pg} / \mathrm{ml})$. Serum Cortisol level was within normal limits. Computed Tomography scan Brain showed wide spread low attenuation areas in bilateral parito-occipital regions and also involving corpus callosum. Magnetic Resonance Imaging brain revealed diffuse symmetrical abnormal MR signals involving bilateral parieto-temporal region through corpus callosum(specially on splenium), suggesting demyelination. MR signals are hypotense on T1W1, nonenhancing and hyper intense on T2W/Flair. The diagnosis of adrenoleukodystrophy was made from history, biochemical and radiological findings. Then treatment was started with prednisolone and phenytoin with control of fits but rest of the symptoms showed no improvement. Bone marrow transplantation is one of the treatment option but he was not suitable for that. Now the child is on medication and physical measure with regular follow-up.

\section{DISCUSSION}

ALD is an autoimmune disorder defined by abnormal accumulation of saturated VLCFA in plasma, brain specially in white matter, testis, skin fibroblast and adrenal cortex which is presented with reduced ability to break fatty acid. ${ }^{5-8}$ The estimated incidence of the disease is $1-5 / 100000 .^{8}$ At present, at least six variants can be distinguished, ${ }^{9}$ they are childhood cerebral ALD, adolescent cerebral ALD, adult cerebral ALD, AMN, the Addison only and asymptomatic phenotype ${ }^{10}$. First neurological manifestations usually appear at 4-8 years of age in the cerebral form of X-ALD. Neurological manifestations include impaired auditory discrimination, visual disturbances, poor coordination, spatialdisorientation, behavorial disorder such as abnormal withdrawl or aggression, poor memory and school performance. Clinical course in adrenoleukodystrophy is charachterized by behavioral dsoreders, visual loss, ataxia, decresed hearing and epileptic seizure followed by mental retardation and death. Adrenal insufficiency is usual finding but does not always manifested by neurologic disease. ${ }^{11}$ Progression usually leads to vegetative state within 2 years. In our patient he is also deteriorating day by day. For diagnosis MRI is more sensitive than CT. Typical demyelination started bilaterally in occipital region but gradually spreads to parietal, temporal and finally frontal region $^{12}$. In our case demyelination found at parieto-occipital region gradually include corpus callosum. Presentation of primary adrenal insufficiency is raised ACTH normal or low cortisol level and serum VLCFA level will be increased. Our case have the same biochemical picture. Prognosis is generally poor and death occur within 
10 years after symptom appear but adult onset is milder. Treatment options of this rare disease is symptomatic. Steriods are used if ther is any adrenal insufficiency, psychotrpics for psychiatric symptom. Lorezo's oil can delay the appearance of cerebral childhood form, it is mixture of oleic acid and erucic acid. Statin also have some role in reducing VLFCA level ${ }^{13}$. Bone marrow transplantation is another option of treatment as it can halt inexorable progressive demyelination and overt neurological manifestation ${ }^{14}$. But it is not done here because of donor rejection. Genetic counselling of family members are advisable. For prevention amniocentesis can done during pregnancy. Lastly rehabilitation is main stream of management as there is not much promise able treatment option. ${ }^{15}$ Combined rehabilitation measures include spasticity management by oral baclofen, Bed positioning by pneumatic bed and pressure mapping, neck control SOMI brace use, supervised exercise like PROM, stretching by physiotherapist, orthosis like AFO, Rolyan figure of eight for sublaxation of shoulder, oromotor stimulation for speech and swallowing rehab start with semisolid food intake training in proper positioning. A diet chart is given which enriched by protein with low- carb and low VLCFA food. Social interaction was done with the help of other family members, relatives and friends.

\section{CONCLUSION}

This a very rare neurological case with poor prognosis is a big challenge to rehabilitate. Our goal is to reduce disability and make condition static and make independent patient as much as possible. Rehabilitation guideline of these type of rare condition will help us to treat such type of illness in future.

\section{ACKNOWLEDGMENT}

The authors are grateful to the Bangabandhu Sheikh Mujib Medical University. Dhaka for providing a grant to conduct this study. Authors also sincerely thank the participants of the study.

\section{REFERENCES}

1. Ulrich J, Herschkowitz N, Heitz P, Sigrist T, Baerlocher P. Adrenoleukodystrophy. Acta neuropathologica. 1978 Jan 1;43(1-2):77-83.

2. Fettes I, Killinger D, Volpe R. Adrenoleukodystrophy: report of a familial case. Clinical endocrinology. 1979 Aug;11(2):151-60.

3. White P C. Adrenocortical insu!ciency. Kliegman RM, Behrman RE, Geme JWS , Schor NF, Stanton BF editors. In : Nelson Textbook of Pediatrics. 19th Ed. Philadelphia : Saunders; 2012. p.1924-6.
4. Schaumburg HH, Powers JM, Raine CS, Suzuki K, Richardson EP. Adrenoleukodystrophy: a clinical and pathological study of 17 cases. Archives of Neurology. 1975 Sep 1;32(9):577-91.

5. Moser HW. Adrenoleukodystrophy: phenotype, genetics, pathogenesis and therapy. Brain. 1989; 120: 1485-1508.

6. Singh I, Moser AB, Goldescher S, Moser HW. Lignoceric acid is oxidized in the peroxisome: implications for the Zellweger cerebro- hepato-renal syndrome and adrenoleukodystrophy. Proc Natl Acad Sci. 1994; 81: 4203-7.

7. Siemerling E, Creutzfeldt HG. Bronzekrankheit und sclerosierende Encephalomyelitis (Di\%use sklerose). Archiv fr Psychiatrie. 1993; 68: 217-44.

8. Mosser J, Douar AM, Sarde CO, et al. Putative X-linked adrenoleukodystrophy gene shares unexpected homology with ABC transporters. Nature. 1993;361: 726-30.

9. Gartner J, Braun A, Holzinger A, Roerig P, Lenard HG, Roscher AA. Clinical and genetic aspects of X-linked adrenoleukodystrophy. Neuropediatrics. 1998; 29(1): $3-13$.

10. Sobue G, Ueno-Natsukari I, Okamoto H. Phenotypic heterogeneity of an adult form of adrenoleukodystrophy in monozygotic twins. Ann Neurol. 1994; 36: 912-915.

11. Castellote A, Vera J, Vazquez E, Roig M, Belmonte JA, Rovira A. MR in adrenoleukodystrophy: atypical presentation as bilateral frontal demyelination. American journal of neuroradiology. 1995 Apr 1;16(4):814-5.

12. Sadeghi-Nejad A, Senior B. Adrenomyeloneuropathy presenting as Addison,s disease in childhood. N Eng J Med. 1990 ; 322: 13-6.

13. Odone A, Odone M. LorenzoÓs oil: a new treatment for adrenoleukodystrophy. J Pediatr Neurosci. 1989; 5: 55-61.

14. Krivit W, Peters C, Shapiro EG. Bone marrow transplantation as effective treatment of central nervous system disease in globoid cell leukodystrophy, metachromatic leukodystrophy, adrenoleukodystrophy, mannosidosis, fucosidosis, aspartylglucosaminuria, Hurler, Maroteaux-Lamy, and Sly syndromes, and Gaucher disease type III. Current opinion in neurology. 1999 Apr 1;12(2):167-76.

15. Renaud DL, Khan S. Development of a multidisciplinary programme for the treatment of X-linked adrenoleukodystrophy. Paediatrics and Child Health. 2009 Dec 1;19:S217-9. 